\title{
On Analysis of Fractional Navier-Stokes Equations via Nonsingular Solutions and Approximation
}

\author{
Emile Franc Doungmo Goufo and Stella Mugisha \\ Department of Mathematical Sciences, University of South Africa, Florida Science Campus, Florida, Gauteng 0003, South Africa \\ Correspondence should be addressed to Emile Franc Doungmo Goufo; dgoufef@unisa.ac.za
}

Received 16 June 2014; Accepted 24 July 2014

Academic Editor: Abdon Atangana

Copyright (C) 2015 E. F. Doungmo Goufo and S. Mugisha. This is an open access article distributed under the Creative Commons Attribution License, which permits unrestricted use, distribution, and reproduction in any medium, provided the original work is properly cited.

\begin{abstract}
Until now, all the investigations on fractional or generalized Navier-Stokes equations have been done under some restrictions on the different values that can take the fractional order derivative parameter $\beta$. In this paper, we analyze the existence and stability of nonsingular solutions to fractional Navier-Stokes equations of type $\left(\mathbf{u}_{t}+\mathbf{u} \cdot \nabla \mathbf{u}+\nabla p-\operatorname{Re}^{-1}(-\nabla)^{\beta} \mathbf{u}=\mathbf{f}\right.$ in $\left.\Omega \times(0, T]\right)$ defined below. In the case where $\beta=2$, we show that the stability of the (quadratic) convergence, when exploiting Newton's method, can only be ensured when the first guess $\mathcal{U}^{0}$ is sufficiently near the solution $\mathcal{U}$. We provide interesting well-posedness and existence results for the fractional model in two other cases, namely, when $1 / 2<\beta<1$ and $\beta \geq(1 / 2)+(3 / 4)$.
\end{abstract}

\section{Introduction}

Navier-Stokes equations (NSEs) are considered as the foundation of fluid mechanics and, strangely enough, are rarely recorded in their entirety. They describe motions of Newtonian fluid flows irrespective of whether they are laminar or turbulent. NSEs are usually obtained according to the following considerations [1].

(i) Constitutive relations governing the fluid are isotropic, which means independent of the orientation of the coordinate system axes.

(ii) At rest the fluid obeys the laws of statics.

(iii) The equation of the fluid is objective; that is, tensors are used. It is independent of the Galilean reference frame in which it is expressed, and independent of the observer.

Although existence and characterization of smooth or nonsingular solutions of NSEs have been comprehensively investigated in numerous works [2-7], they remain open problems. Fractional calculus applied to differential equations has captured a huge amount of attention recently [8-12] and it was shown that its applications lie in modeling many problems and phenomena in applied technology, engineering, and sciences including applied mathematics, computing, physics, biology, chemistry, economic, and other domains of applications.

\section{A Brief Overview on Fractional Order Derivatives}

There is a huge amount of works concerning various definitions of fractional derivatives, but two of them have emerged as the most popular and most applicable on dynamical systems: the Caputo derivative and Riemann-Liouville derivative. Caputo derivative is expressed as

$$
\begin{aligned}
{ }_{0}^{C} D_{x}^{\beta}(u(x)) & =\frac{\partial^{\beta}}{\partial x^{\beta}} u(x) \\
& =\frac{1}{\Gamma(n-\beta)} \int_{0}^{x}(x-t)^{n-\beta-1} \frac{d^{n} u(t)}{d t^{n}} d t .
\end{aligned}
$$


For the fractional derivative in the sense of RiemannLiouville, we have the following definition:

$$
\begin{aligned}
D_{x}^{\beta}(u(x)) & =\frac{\partial^{\beta}}{\partial x^{\beta}} u(x) \\
& =\frac{1}{\Gamma(n-\beta)} \frac{d^{n}}{d t^{n}} \int_{0}^{x}(x-t)^{n-\beta-1} u(t) d t .
\end{aligned}
$$

In applied sciences in general and dynamical system in particular, each of these fractional derivatives presents some advantages and disadvantages $[8,13]$. For example, Caputo derivatives are defined only for differentiable functions while functions that have no first order derivative might have fractional derivatives of all orders less than one in the sense of Riemann-Liouville [11]. Another example is the fact that the Riemann-Liouville derivative of a constant is not zero unlike Caputo's derivative which gives zero when applying to a constant function. However Caputo's derivative demands higher conditions of regularity for differentiability. Then, to compute the fractional derivative of a function in the Caputo sense, we have to calculate first its derivative. Fortunately for Riemann-Liouville derivative, the author in [14] proposed the following alternative definition:

$$
\begin{aligned}
D_{x}^{\beta}(u(x)) & =\frac{\partial^{\beta}}{\partial x^{\beta}} u(x) \\
& =\frac{1}{\Gamma(n-\beta)} \frac{d^{n}}{d t^{n}} \int_{0}^{x}(x-t)^{n-\beta-1}\{u(t)-u(0)\} d t
\end{aligned}
$$

the above modified Riemann-Liouville derivative seems to have advantages of both the standard Riemann-Liouville and Caputo fractional derivatives: it is defined for arbitrary continuous (nondifferentiable) functions and the fractional derivative of a constant is equal to zero. However from its definition we do not actually give a fractional derivative of a function say $u(x)$ but the fractional derivative of $u(x)-$ $u(0)$ can lead to fractional derivative that is not defined at the origin for some function for which $u(0)$ does not exist. We note that Caputo and Riemann-Liouville may have their disadvantages but still remain the best definition of the fractional derivative according to their easy applicability. We need the following definition.

Definition 1 (partial derivatives of fractional order). Assume now that $u(x)$ is a function of $n$ variables $x_{i}, i=1, \ldots, n$ also of class $C$ on $D \in \mathbb{R}_{n}$. We define as partial derivative of order $\beta$ for $u(x)$ with respect to $x_{i}$ the function

$$
a \partial_{\underline{x}}^{\beta} u=\left.\frac{1}{\Gamma(m-\beta)} \int_{a}^{x_{i}}\left(x_{i}-t\right)^{m-\beta-1} \partial_{x_{i}}^{m} u\left(x_{j}\right)\right|_{x_{j}} d t
$$

if it exists, where $\partial_{x_{i}}^{m}$ is the usual partial derivative of integer order $m$.

In this paper, we consider the fractional derivative $(-\nabla)^{\beta}$ in the sense of Caputo to obtain and analyze the generalized (fractional) NSE in 3D of the form

$$
\mathbf{u}_{t}+\mathbf{u} \cdot \nabla \mathbf{u}+\nabla p-\operatorname{Re}^{-1}(-\nabla)^{\beta} \mathbf{u}=\mathbf{f} \quad \text { in } \Omega \times(0, T],
$$

with $\beta>0$ a real number. The system is subject to the incompressibility condition,

$$
\nabla \cdot \mathbf{u}=0 \quad \text { in } \Omega \times(0, T]
$$

the homogeneous no-slip boundary condition,

$$
\mathbf{u}=0 \quad \text { on } \Gamma_{\text {rigid }} \times(0, T],
$$

the inflow condition

$$
\mathbf{u}^{\text {in }}=\mathbf{u} \quad \text { on } \Gamma_{\text {in }},
$$

and the initial condition,

$$
\mathbf{u}(\mathbf{x}, 0)=\mathbf{u}_{0}(\mathbf{x}) \quad \text { in } \Omega,
$$

where $\Omega \subseteq \mathbb{R}^{3}, \mathbf{u}=(u, v, w)=(u(t, x, y, z), v(t, x, y, z)$, $w(t, x, y, z))$ is the fluid velocity vector field with components $u, v$, and $w$ at the point $(x, y, z)$ and time $t$. Note that $x=x(t)$, $y=y(t), z=z(t), u=d x / d t, v=d y / d t$, and $w=d z / d t$. Here $\mathbf{f}$ is the body force per unit mass (note that we will assume that $\mathbf{f}=0$ ). $\Gamma_{\text {rigid }}$ and $\Gamma_{\text {in }}$ are the rigid part and the inflow part of the boundary $\Gamma$, respectively.

\section{Classical Model of Navier-Stokes Equations}

3.1. Model Description and Definitions. The theory of mechanics of continuous media, also known as continuum mechanics, allows the description of the constitutive equations laws that describe the deformations of fluid medium. These laws, in combination with the general conservation principles (conservation of mass and of momentum), form the system of partial differential equations, which are equal in number to the number of unknowns of the system. Namely, for 3D motion there are four dependent variables: $u, v, w$, and $p$, and four independent variables: $x, y, z$, and $t$. This leads to the following classical Navier-Stokes equations, obtained from the generalized version (5) by putting $\beta=2$, and of which we discuss a possible treatment using the method of finite elements technique:

$$
\mathbf{u}_{t}+\mathbf{u} \cdot \nabla \mathbf{u}+\nabla p-\operatorname{Re}^{-1} \nabla^{2} \mathbf{u}=\mathbf{f} \quad \text { in } \Omega \times(0, T],
$$

with the incompressibility condition, boundary condition, inflow condition, and initial condition remaining the same as (6), (7), (8), and (9), respectively. In addition we assume that $\Omega$ does not change in time.

The finite element discretization of the Navier-Stokes problem is based on the variational formulation, and the use of Sobolev spaces is needed for the mathematical treatment of the variational formulation of the model. We use subspaces of the usual Hilbert space

$$
L^{2}(\Omega)=\left\{f: \int_{\Omega}|f|^{2} d \mathbf{x}<\infty\right\}
$$

of square-integrable functions on $\Omega$, where integration is in the sense of Lebesgue:

$$
L_{0}^{2}(\Omega)=\left\{f: f \in L^{2}(\Omega),(f, 1)=0\right\}
$$


and the corresponding inner products and norms

$$
(f, g)=\int_{\Omega} f g d \mathbf{x}, \quad\|f\|_{0}=(f, f)^{1 / 2} .
$$

Next, for any nonnegative integer $k$, we define the Sobolev space

$$
H^{k}(\Omega)=\left\{f: f \in L^{2}(\Omega), D^{s} f \in L^{2}(\Omega), \text { for } s=1, \ldots, k\right\}
$$

of square-integrable functions, all of whose derivatives of order up to $k$ are also square integrable, where $D^{s}$ denotes any and all derivatives of order $s . H^{k}(\Omega)$ comes with the norm

$$
\|f\|_{k}=\left(\|f\|_{0}^{2}+\sum_{s \leq k, s \neq 0}\left\|D^{s} f\right\|_{0}^{2}\right)^{1 / 2} .
$$

The following definitions can now be stated:

$$
\begin{gathered}
H^{0}(\Omega)=L^{2}(\Omega) \\
H^{1}(\Omega)=\left\{f: f \in L^{2}(\Omega), \partial_{i} f \in L^{2}(\Omega), 1 \leq i \leq 3\right\} \\
\|\nabla f\|_{0}=(\nabla f, \nabla f)^{1 / 2} \\
\|f\|_{1}=\left(\|f\|_{0}^{2}+\|\nabla f\|_{0}^{2}\right)^{1 / 2}=\left(\|f\|_{0}^{2}+\sum_{i=1}^{3}\left\|\frac{\partial f}{\partial x_{i}}\right\|_{0}^{2}\right)^{1 / 2} .
\end{gathered}
$$

Of particular interest is the subspace of $H_{0}^{1}(\Omega)$ of $H^{1}(\Omega)$ defined by

$$
H_{0}^{1}(\Omega)=\left\{f: f \in H^{1}(\Omega), f=0 \text { on } \Gamma\right\},
$$

whose elements vanish on the boundary $\Gamma$.

For functions belonging to $H^{1}(\Omega)$, the seminorm

$$
|f|_{1}=\left(\sum_{i=1}^{3}\left\|\frac{\partial f}{\partial x_{i}}\right\|_{0}^{2}\right)^{1 / 2}
$$

defines a norm equivalent to (16). The proof of this statement is not our aim in this dissertation, but it can be found in Braess [15]. Thus for such functions, (18) may be used instead of (16).

We denote by $H^{-1}(\Omega)$ the dual space consisting of bounded linear functionals on $H_{0}^{1}(\Omega)$; that is, $f \in H^{-1}(\Omega)$ implies that $(f, w) \in \mathbb{R}$ for all $w \in H_{0}^{1}(\Omega)$. A norm for $H^{-1}(\Omega)$ is given by

$$
\|f\|_{-1}=\sup _{0 \neq w \in H_{0}^{1}(\Omega)} \frac{(f, w)}{|w|_{1}} .
$$

Since the velocity field $\mathbf{u}=\mathbf{u}(u, v, w)=\left(\mathbf{u}_{i}\right)_{i=1,2,3}$ is a vector valued function, we use the spaces

$$
\begin{aligned}
\mathbf{H}^{k}(\Omega) & =H^{k}(\Omega)^{3}=\left\{\mathbf{u}: \mathbf{u}_{i} \in H^{k}(\Omega) \text { for } i=1,2,3\right\}, \\
\mathbf{H}_{0}^{1}(\Omega) & =H_{0}^{1}(\Omega)^{3}=\left\{\mathbf{u}: \mathbf{u}_{i} \in H_{0}^{1}(\Omega) \text { for } i=1,2,3\right\}, \\
\mathbf{H}^{-1}(\Omega) & =H^{-1}(\Omega)^{3}=\left\{\mathbf{u}: \mathbf{u}_{i} \in H^{-1}(\Omega) \text { for } i=1,2,3\right\} .
\end{aligned}
$$

For $k \geq 0, \mathbf{H}^{k}(\Omega)$ is equipped with the norm

$$
\|\mathbf{u}\|_{k}=\left(\sum_{i=1}^{3}\left\|\mathbf{u}_{i}\right\|_{k}^{2}\right)^{1 / 2} .
$$

Alternatively, for functions belonging to $\mathbf{H}_{0}^{1}(\Omega)$, we may use

$$
|\mathbf{u}|_{1}=\left(\sum_{i=1}^{3}\left|\mathbf{u}_{i}\right|_{1}^{2}\right)^{1 / 2}
$$

The inner product for functions belonging to $\mathbf{L}^{2}(\Omega)=$ $\mathbf{H}^{0}(\Omega)=L^{2}(\Omega)^{3}$ is also given by

$$
(\mathbf{u}, \mathbf{w})=\int_{\Omega} \mathbf{u} \cdot \mathbf{w} d \mathbf{x} .
$$

Before stating the weak variational formulation for our model, let us discuss some preliminaries concerning the existence and uniqueness of a solution of the Navier-Stokes equations.

3.2. Existence and Uniqueness for a Solution of Navier-Stokes Equations. From the mathematical point of view, two questions concerning the Navier-Stokes equations are of main interest. Given a set of data which are sufficiently smooth,

(1) does a solution of (10), (6)-(9) exist?

(2) if a solution exists, is it unique?

First, we have to clarify the notion of a solution of (10), (6)-(9). There exist several concepts of the notion of a solution of the above system, the most important of which are the classical solution and the weak solution.

Definition 2 (classical solution). A pair $(\mathbf{u}, p)$ is called a classical solution of the Navier-Stokes problem (10), (6)-(9) if

(1) $(\mathbf{u}, p)$ satisfies the Navier-Stokes problem (10), (6)(9),

(2) $\mathbf{u}$ and $p$ are infinitely many times differentiable with respect to space and time variables.

Then, according to John and Kaya [16], the existence of a classical solution of (10), (6)-(9) cannot yet be proven, but if a classical solution exists, it is unique.

To define a weak solution, we first need to transform (10) into a weak form by

(i) multiplying (10) with a suitable vector valued function $\varphi$ (test function),

(ii) integrating over $\Omega \times(0, T]$,

(iii) applying integration by parts (Green's theorem).

The last step is possible only if there are some restrictions on the domain. For the test function $\varphi$, one requires

(i) $\varphi \in C_{0, \operatorname{div}}^{\infty}(\Omega)$ for each time $t$, where $C_{0, \operatorname{div}}^{\infty}(\Omega)=\{\mathbf{f}$ : $\left.\mathbf{f} \in C_{0}^{\infty}(\Omega), \nabla \cdot \mathbf{f}=0\right\}$, 
(ii) $\varphi$ is infinitely differentiable with respect to time,

(iii) $\varphi(\cdot, T)=\mathbf{0}$.

This gives the weak formulation of the Navier-Stokes equations

$$
\begin{gathered}
\int_{0}^{T}\left[-\left(\mathbf{u}, \varphi_{t}\right)+(\mathbf{u} \cdot \nabla \mathbf{u}, \varphi)+\operatorname{Re}^{-1}(\nabla \mathbf{u}, \nabla \varphi)\right] d t \\
=\int_{o}^{T}(\mathbf{f}, \varphi) d t+\left(\mathbf{u}_{0}, \varphi(\cdot, 0)\right),
\end{gathered}
$$

which has the following features:

(i) there is no time derivative of $\mathbf{u}$,

(ii) there is no second order spatial derivative with respect to $\mathbf{u}$,

(iii) the pressure vanishes, since Green's formula yields

$$
(\nabla p, \varphi)=\int_{\partial \Omega} p \varphi \cdot \mathbf{n} d s-(p, \nabla \cdot \varphi)=0
$$

because $\varphi \cdot \mathbf{n}=0$ on $\partial \Omega$ and $\nabla \cdot \varphi=0$.

Definition 3 (weak solution). A function $\mathbf{u}$ is called weak solution of the Navier-Stokes equations if

(1) $\mathbf{u}$ satisfies (24) for all test functions $\varphi$ with the properties on $\varphi$ given above,

(2) $\mathbf{u}$ has the following regularity:

$$
\mathbf{u} \in L^{2}\left(0, T ; H_{0, \operatorname{div}}^{1}(\Omega)\right) \cap L^{\infty}\left(0, T ; L_{\text {div }}^{2}(\Omega)\right),
$$

where the subscript div means space of divergence-free functions; for instance,

$$
\begin{gathered}
C_{0, \operatorname{div}}^{\infty}(\Omega)=\left\{\mathbf{f}: \mathbf{f} \in C_{0}^{\infty}(\Omega), \nabla \cdot \mathbf{f}=0\right\}, \\
L^{2}\left(0, T ; H_{0}^{1}(\Omega)\right)=\left\{f(\mathbf{x}, t): \int_{0}^{T}\|f\|_{0}^{2} d t<\infty\right\} .
\end{gathered}
$$

More generally

$$
\begin{aligned}
L^{q} & \left(t_{0}, t_{1} ; X\right) \\
& =\left\{f(\mathbf{x}, t): \int_{t_{0}}^{t_{1}}\|f\|_{X}^{q} d t<\infty\right\} \text { for any } q \in[1, \infty)
\end{aligned}
$$

is the space of strongly measurable maps $f:\left[t_{o}, t_{1}\right] \rightarrow X$, such that

$$
\|f\|_{L^{q}\left(t_{0}, t_{1} ; X\right)}=\left(\int_{t_{0}}^{t_{1}}\|f\|_{X}^{q} d t\right)^{1 / q}<\infty \text { for } q \in[1, \infty)
$$

and $X$ is a Banach space. Furthermore

$$
L^{\infty}\left(t_{0}, t_{1} ; X\right)=\left\{f(\mathbf{x}, t): \text { ess } \sup _{t_{0} \leq t \leq t_{1}}\|f\|_{X}<\infty\right\}
$$

with

$$
\|f\|_{L^{\infty}\left(t_{0}, t_{1} ; X\right)}=\operatorname{ess} \sup _{t_{0} \leq t \leq t_{1}}\|f\|_{X}<\infty \quad \text { for } q=\infty .
$$

It is obvious that all these spaces are needed for the weak formulation given in the next section.
The existence of a weak solution of (10), (6)-(9) was proved in 1934 by Leray [17]. The weak solution is unique if every other weak solution satisfies an additional regularity assumption, Serrin's condition; see Serrin [7] or John and Kaya [16]. But it is not known in 3D if every weak solution possesses such additional condition.

According to the same article [16], the existence of a weak solution of the Navier-Stokes equations can be proven in arbitrary domains, but the uniqueness cannot yet be proven.

The answer to the question of uniqueness of the weak solution in $3 D$ or existence of a classical solution in $3 D$ is one of the major mathematical challenges of this century (John and Kaya [16]). There is a prize of one million US Dollars for people who can answer these questions.

3.3. The Stationary Case. We now consider our domain $\Omega$ defined in the first chapter and assume that all the variables in the system (10), (6)-(9) are independent of time. We therefore obtain the following stationary Navier-Stokes problem:

$$
\begin{gathered}
\mathbf{u} \cdot \nabla \mathbf{u}+\nabla p-\operatorname{Re}^{-1} \nabla^{2} \mathbf{u}=\mathbf{f}, \\
\nabla \cdot \mathbf{u}=0, \quad \text { in } \Omega
\end{gathered}
$$

with the following boundary conditions:

$$
\left.\mathbf{u}\right|_{\Gamma_{\text {rigid }}}=0,\left.\quad \mathbf{u}\right|_{\Gamma_{\text {in }}}=\mathbf{u}^{\text {in }}
$$

having the following weak formulation.

Find functions $\mathbf{u} \in \mathbf{u}^{\text {in }}+\mathbf{H}_{0}^{1}(\Omega)$ and $p \in L_{0}^{2}(\Omega)$ such that

$$
\begin{gathered}
a(\mathbf{u}, \mathbf{v})+c(\mathbf{u}, \mathbf{u}, \mathbf{v})+b(p, \mathbf{v})=(\mathbf{f}, \mathbf{v}) \quad \forall \mathbf{v} \in \mathbf{H}_{0}^{1}(\Omega) \\
b(q, \mathbf{u})=0 \quad \forall q \in L_{0}^{2}(\Omega),
\end{gathered}
$$

discretized as follows.

Find a pair $\left(\mathbf{u}^{h}, p^{h}\right) \in \mathbf{u}^{\text {in }}+\mathbf{V}_{0}^{h} \times S_{0}^{h}$ such that

$$
\begin{array}{rr}
a\left(\mathbf{u}^{h}, \mathbf{v}^{h}\right)+c\left(\mathbf{u}^{h}, \mathbf{u}^{h}, \mathbf{v}^{h}\right)+b\left(p^{h}, \mathbf{v}^{h}\right) & =\left(\mathbf{f}, \mathbf{v}^{h}\right) \\
& \forall \mathbf{v}^{h} \in \mathbf{V}_{0}^{h} \\
b\left(q^{h}, \mathbf{u}^{h}\right)=0 \quad \forall q^{h} \in S_{0}^{h} . &
\end{array}
$$

For the sake of a stable approximation as $h \rightarrow 0$, it is crucial that we relate the continuous and discrete spaces by the following hypotheses.

Hypothesis $\mathbf{H} \mathbf{1}$ (approximation property of $\mathbf{V}_{0}^{h}$ ). There exists an operator $r^{h} \in \mathscr{L}\left(\left[H^{2}(\Omega) \cap H_{o}^{1}(\Omega)\right]^{2} ; \mathbf{V}_{0}^{h}\right)$ and an integer $l$ such that

$$
\left\|\varphi-r^{h} \varphi\right\|_{1} \leq C h^{m}\|\varphi\|_{m+1} \quad \forall \varphi \in \mathbf{H}^{m+1}(\Omega), \quad 1 \leq m \leq l .
$$

Hypothesis $\mathbf{H} \mathbf{2}$ (approximation property of $Q^{h}$ ). There exists an operator $s^{h} \in \mathscr{L}\left(L^{2}(\Omega) ; Q^{h}\right)$ such that

$$
\left\|q-s^{h} q\right\|_{0} \leq C h^{m}\|q\|_{m} \quad \forall q \in H^{m}(\Omega), \quad 0 \leq m \leq l .
$$


Hypothesis $\mathbf{H 3}$ (uniform inf-sup condition ). For each $q^{h} \in S_{0}^{h}$, there exists a $\mathbf{v}^{h} \in \mathbf{V}_{0}^{h}$ such that

$$
\begin{gathered}
b\left(q^{h}, \mathbf{v}^{h}\right)=\left\|q^{h}\right\|_{0}^{2} \\
\left|\mathbf{v}^{h}\right|_{1} \leq C\left\|q^{h}\right\|_{0},
\end{gathered}
$$

where the constant $C>0$ is independent of $h, q^{h}$ and $v^{h}$; $\mathscr{L}(Y, W)$ is the space of linear operators from $Y$ to $W ;\|\cdot\|_{0}$ and $\|\cdot\|_{m}$ are the standard norms in $L^{2}(\Omega)$ and $H^{m}(\Omega)$, respectively; $|\cdot|_{1}$ is the standard seminorm in $H^{1}(\Omega)$.

We assume that the bilinear forms $a(\cdot, \cdot)$ and $b(\cdot, \cdot)$ and the approximating subspaces $\mathbf{V}_{0}^{h}$ and $S_{0}^{h}$ satisfy all the hypotheses H1, H2, H3 required for suitable approximations. For any f $\in \mathbf{H}^{-1}(\Omega)$, the system (35) has a solution $\left(\mathbf{u}^{h}, p^{h}\right)$. It is well known (see Girault and Raviart [4] or Gunzburger [18]) that the solution is unique for "sufficiently small" data $\mathbf{f}$ or "sufficiently small" Reynolds number, Re. In fact if we set.

$$
\mathbf{Z}=\left\{\mathbf{v} \in \mathbf{H}_{0}^{1}(\Omega): b(q, \mathbf{v})=0 \quad \forall q \in L_{0}^{2}(\Omega)\right\}
$$

the space of divergence-free functions, and

$$
\mathcal{N}=\sup _{\mathbf{u}, \mathbf{v}, \mathbf{w} \in \mathbf{Z}} \frac{c(\mathbf{w}, \mathbf{u}, \mathbf{v})}{|\mathbf{u}|_{1}|\mathbf{v}|_{1}|\mathbf{w}|_{1}}
$$

then given $\mathbf{f} \in \mathbf{H}^{-1}(\Omega)$, and if $(\operatorname{Re})^{2} \mathcal{N}\|\mathbf{f}\|_{-1}<1$, then the problem (34) has a unique solution $(\mathbf{u}, p) \in \mathbf{Z} \times L_{0}^{2}(\Omega)$.

From the previous assertion, we can state one of the fundamental properties (see Joseph [5]) of the solutions of the Navier-Stokes equations (32) and (33) which reads as follows: when the viscosity is large (or the Reynolds number is small), all solutions of the Navier-Stokes equations tend to a single basic flow. So what is the final destiny of all these uniquely determined solutions of the Navier-Stokes equations? For large values of the Reynolds number, the final set of flows which evolve from a given set of initial fields is generally "turbulent."

As just proven, the Navier-Stokes equations have in general more than one solution, unless the data satisfies very stringent requirements. However, it can also be shown that in many practical examples these solutions are mostly isolated; that is, there exists a neighborhood in which each solution is unique. Furthermore, it can be established (Girault and Raviart [4]) that the solutions depend continuously on the Reynolds number, Re (which is inversely proportional to the kinematic viscosity). Thus as the Reynolds number varies along an interval, each solution of the Navier-Stokes equations describes an isolated branch. In particular, this means that the bifurcation phenomenon can be rare or occurs infrequently (Girault and Raviart [4]). This situation, frequently encountered in practice, is expressed mathematically by the notion of branches of nonsingular solutions. So the solutions of the problem (32) and (33) are "in general" nonsingular.

But when the Reynolds number Re is large (small viscosity), compared to the other parameters of the fluid, there arises a boundary layer in the neighborhood of $\partial \Omega$ where the viscosity predominates while it is negligible in the interior of $\Omega$. At the same time, the transition to turbulence occurs. Thus the solutions of the Navier-Stokes equations are seriously discontinuous at certain values of the Reynolds number, Re.

In the coming sections, we show that, under hypotheses $\mathbf{H 1}, \mathbf{H 2}$, H3, the problem (35) possesses a branch of nonsingular solutions that, as $h \rightarrow 0$, converges to a given branch of nonsingular solutions of (34). This convergence depends on the Reynolds number Re. We also use a finite element method, the Newton method, to show that the stability of such a convergence is guaranteed for small variations of Re.

3.4. Nonsingular Solutions and Approximation. Let $Z$ and $\mathscr{Z}$ be two Banach spaces and $\Lambda$ a compact interval of the real line $\mathbb{R}$. We are given a $\mathscr{C}^{p}$-mapping $(p \geq 1)$

$$
F:(\operatorname{Re}, \mathcal{U}) \in \Lambda \times Z \longrightarrow F(\operatorname{Re}, \mathcal{U}) \in \mathscr{Z}
$$

and we want to solve the equation

$$
F(\operatorname{Re}, \mathcal{U})=0 ;
$$

that is, we want to find pairs $(\operatorname{Re}, \mathcal{U}) \in \Lambda \times Z$ which are solutions of (42).

Let $\{(\operatorname{Re}, \mathcal{U}(\operatorname{Re})) ; \operatorname{Re} \in \Lambda\}$ be a branch of solutions of (42). This means that

$\operatorname{Re} \longrightarrow \mathcal{U}(\mathrm{Re})$ is a continuous function from $\Lambda$ into $Z$,

$$
F(\operatorname{Re}, \mathcal{U}(\operatorname{Re}))=0 .
$$

Moreover, we suppose that these solutions are nonsingular in the sense that

$D_{\mathscr{U}} F(\mathrm{Re}, \mathcal{U}(\mathrm{Re}))$ is an isomorphism from $Z$ onto $\mathscr{Z}$

$\forall \operatorname{Re} \in \Lambda$.

As an immediate consequence of (44), it follows from the implicit function theorem (see [4]) that $\operatorname{Re} \rightarrow \mathscr{U}(\mathrm{Re})$ is a $\mathscr{C}^{P}$-function from $\Lambda$ into $Z$.

Let us show that our problem for the Navier-Stokes equations (34) fits into the above framework. We first set

$$
Z=\mathscr{Z}=\mathbf{H}^{1}(\Omega) \times L_{0}^{2}(\Omega),
$$

and we introduce the intermediate space

$$
Y=\mathbf{H}^{-1}(\Omega) \times\left\{g \in \mathbf{H}^{1 / 2}(\Gamma) ; \int_{\Gamma} g \cdot \mathbf{n} d x=0\right\} .
$$

Next we define a linear operator $T$ as follows: given $\left(\mathbf{f}_{*}, g_{*}\right) \in$ $Y$, we denote by $\left(\mathbf{u}_{*}, p_{*}\right)=T\left(\mathbf{f}_{*}, g_{*}\right) \in Z$ the solution of the Dirichlet problem for the Stokes equations:

$$
\begin{gathered}
-\nabla^{2} \mathbf{u}_{*}+\nabla p_{*}=\mathbf{f}_{*} \quad \text { in } \Omega \\
\nabla \cdot \mathbf{u}_{*}=0 \quad \text { in } \Omega \\
\left.\mathbf{u}_{*}\right|_{\Gamma}=g_{*} .
\end{gathered}
$$


Finally, with the data $(\mathbf{f}, g) \in Y$, we associate a $\mathscr{C}^{\infty}$-mapping $G$ from $\mathbb{R}_{+} \times Z$ into $Y$, defined for a $\mathscr{U}=(\mathbf{w}, q) \in Z$, by

$$
G:(\operatorname{Re}, \mathcal{U}) \longrightarrow G(\operatorname{Re}, \mathscr{U})=(\operatorname{Re}(\mathbf{w} \cdot \nabla \mathbf{w}-\mathbf{f}),-g)
$$

and we set

$$
F(\operatorname{Re}, \mathcal{U})=\mathscr{U}+T G(\operatorname{Re}, \mathcal{U}) .
$$

It is clear that $(\mathbf{f}, g)=(\mathbf{f}, 0) \in Y$ and (48) becomes

$$
G(\operatorname{Re}, \mathcal{U})=(\operatorname{Re}(\mathbf{w} \cdot \nabla \mathbf{w}-\mathbf{f}), 0)
$$

or simply

$$
G(\operatorname{Re}, \mathcal{U})=\operatorname{Re}(\mathbf{w} \cdot \nabla \mathbf{w}-\mathbf{f}) .
$$

Now we may state the lemma.

Lemma 4. The pair $(\mathbf{u}, p) \in \mathbf{H}^{1}(\Omega) \times L_{0}^{2}(\Omega)$ is a solution of problem (32) and (33) if and only if (Re, $\mathcal{U}$ ), with $\mathscr{U}=$ (u, $\operatorname{Re} p$ ), is a solution of (42), where the spaces $Z$ and $\mathscr{Z}$ are defined by (45) and the compound mapping $F$ is defined by (49) and (50).

Proof. If $(\mathbf{u}, p)$ is a solution of the problem (32) and (33) then

$$
\begin{gathered}
-\nabla^{2} \mathbf{u}+\nabla(\operatorname{Re} p)=\operatorname{Re}(\mathbf{f}-\mathbf{u} \cdot \nabla \mathbf{u}) \quad \text { in } \Omega \\
\nabla \cdot \mathbf{u}=0 \quad \text { in } \Omega \\
\mathbf{u}=0 \quad \text { on } \Gamma_{\text {rigid }} .
\end{gathered}
$$

From both (47) and $\left(\mathbf{u}_{*}, p_{*}\right)=T\left(f_{*}, g_{*}\right)$, applied to $f_{*}=$ $\operatorname{Re}(\mathbf{f}-\mathbf{u} \cdot \nabla \mathbf{u})$ and $p_{*}=\operatorname{Re} p$, we can write

$$
(\mathbf{u}, \operatorname{Re} p)=T(\operatorname{Re}(\mathbf{f}-\mathbf{u} \cdot \nabla \mathbf{u}), 0)
$$

or

$$
(\mathbf{u}, \operatorname{Re} p)-T(\operatorname{Re}(\mathbf{f}-\mathbf{u} \cdot \nabla \mathbf{u}), 0)=0
$$

(since the operator $T$ is linear).

Then, (51) yields

$$
\mathcal{U}+T G(\operatorname{Re}, \mathcal{U})=0
$$

and (49) yields

$$
F(\operatorname{Re}, \mathcal{U})=0 \text {, }
$$

which means

$$
\text { (Re, } \mathscr{U}) \text { is a solution of (39). }
$$

From Lemma 4, it is clear that if $(\mathbf{u}, p)$ is a solution of the problem (32) and (33), then $(\operatorname{Re}, \mathcal{U})$, where $\mathcal{U}=(\mathbf{u}, \operatorname{Re} p)$, is a nonsingular solution of (42).

We may now state the following proposition.
Proposition 5. Assume that hypotheses $\mathbf{H 1}, \mathbf{H} 2$, and $\mathbf{H} 3$ hold. Let $\{(\operatorname{Re},(\mathbf{u}(\operatorname{Re}), \operatorname{Re} p(\operatorname{Re})))$; Re $\in \Lambda\}$ be a branch of nonsingular solutions of the Navier-Stokes problem (34). Then there exists a neighborhood $\mathcal{O}$ of the origin in $\mathbf{H}_{0}^{1}(\Omega) \times L_{0}^{2}(\Omega)$ and for $h \leq h_{0}$ sufficiently small a unique $\mathscr{C}^{\infty}$ branch $\left\{\left(\operatorname{Re},\left(\mathbf{u}^{h}(\operatorname{Re}), \operatorname{Re} p^{h}(\mathrm{Re})\right)\right) ; \operatorname{Re} \in \Lambda\right\}$ of nonsingular solutions of problem (35) such that

$$
\begin{array}{r}
\left(\mathbf{u}^{h}(\operatorname{Re}), \operatorname{Re} p^{h}(\operatorname{Re})\right) \in(\mathbf{u}(\operatorname{Re}), \operatorname{Re} p(\operatorname{Re}))+\mathcal{O} \\
\forall \operatorname{Re} \in \Lambda .
\end{array}
$$

Moreover, we have the convergence property

$$
\limsup _{h \rightarrow 0 \operatorname{Re} \in \Lambda}\left\{\left|\mathbf{u}^{h}(\operatorname{Re})-\mathbf{u}(\operatorname{Re})\right|_{1}+\left\|p^{h}(\operatorname{Re})-p(\operatorname{Re})\right\|_{0}\right\}=0 .
$$

In addition, if the mapping $\mathrm{Re} \rightarrow(\mathbf{u}(\mathrm{Re}), p(\mathrm{Re}))$ is continuous from $\Lambda$ into $H^{m+1}(\Omega)^{3} \times H^{m}(\Omega)$ for some integer $m$ with $1 \leq m \leq l$, we have for all $\operatorname{Re} \in \Lambda$

$$
\begin{aligned}
& \left|\mathbf{u}^{h}(\mathrm{Re})-\mathbf{u}(\mathrm{Re})\right|_{1}+\left\|p^{h}(\mathrm{Re})-p(\mathrm{Re})\right\|_{0} \\
& \leq K h^{m} .
\end{aligned}
$$

Our goal is not to prove this proposition, but to use the results. A similar proof of this proposition can be found in Girault and Raviart [4].

It is also possible to derive an $L^{2}$-estimate for the velocity. But the following regularity must be satisfied:

The mapping $(\phi, \mu) \longmapsto \nabla \mu-\operatorname{Re}^{-1} \nabla^{2} \phi$ is an isomorphism

$$
\text { from }\left[\mathbf{H}^{2}(\Omega) \cap \mathcal{S}\right] \times\left[H^{1}(\Omega) \cap L_{0}^{2}(\Omega)\right] \text { onto } \mathbf{L}^{2}(\Omega) \text {, }
$$

where $\mathcal{S}$ is a closed subspace of $Z$.

Proposition 6. We retain the hypotheses of Proposition 5 and we assume that (61) holds. If the mapping $\mathrm{Re} \rightarrow(\mathbf{u}(\mathrm{Re})$, $p(\mathrm{Re}))$ is continuous from $\Lambda$ into $\mathbf{H}^{m+1}(\Omega) \times H^{m}(\Omega)$ for some integer $m \in[1, l]$, then we have the following $L^{2}$-estimate for all $\operatorname{Re} \in \Lambda$ :

$$
\left\|\mathbf{u}^{h}(\mathrm{Re})-\mathbf{u}(\mathrm{Re})\right\|_{0} \leq K h^{m+1} .
$$

These propositions show that the convergence of finite element approximations is guaranteed, and the stability constants depend on Re.

3.5. Stability of Newton's Method. The method discussed here is one of the finite element methods that is intended to solve the Navier-stokes equations. We saw in Lemma 4 that it suffices to investigate equations of type (42):

$$
F(\operatorname{Re}, \mathcal{U})=0 \text {, }
$$


where $F$ is defined by (49) and is a $\mathscr{C}^{P}$-mapping $(P \geq 1)$ defined on $\Lambda \times Z$ (with $\Lambda$ a compact interval of the real line $\mathbb{R}$ and $\left.Z=\mathbf{H}^{1}(\Omega) \times L_{0}^{2}(\Omega)\right)$. For simplicity, let us set and fix

$$
\lambda=\operatorname{Re} \in \Lambda
$$

and assume that $\mathscr{U}=\mathscr{U}(\lambda)$ is a nonsingular solution of (63). Then

$$
F(\lambda, \mathcal{U})=0,
$$

$D_{\mathscr{U}} F(\lambda, \mathcal{U})$ is an isomorphism from $Z$ onto $\mathscr{Z}$,

where $\mathscr{Z}$ is a Banach space.

Since $\mathcal{U}$ is an isolated solution of (63) and since $F$ is at least differentiable, an efficient way to approximate $\mathcal{U}$ is by the Newton method. The Newton algorithm reads as follows.

Starting from an initial guess $\mathcal{U}^{0}$, construct the sequence $\left\{\mathscr{U}^{n}\right\}_{n}$ in $Z$ by

$$
\mathcal{U}^{n+1}=\mathcal{U}^{n}-\left[D_{\mathcal{U}} F\left(\lambda, \mathscr{U}^{n}\right)\right]^{-1} \cdot F\left(\lambda, \mathscr{U}^{n}\right) \quad n \geq 0
$$

or equivalently

$$
D_{\mathcal{U}} F\left(\lambda, \mathcal{U}^{n}\right) \cdot\left(\mathcal{U}^{n+1}-\mathcal{U}^{n}\right)=-F\left(\lambda, \mathcal{U}^{n}\right) .
$$

As $D_{\mathscr{U}} F(\lambda, \mathcal{U})$ is a linear operator, each step of Newton's method requires the solution of a different problem relative to $D_{\mathscr{U}} F\left(\lambda, \mathscr{U}^{n}\right)$. If this is too costly, the simplest alternative is to replace (66) by

$$
\mathcal{U}^{n+1}=\mathscr{U}^{n}-\left[D_{\mathcal{U}} F\left(\lambda, \mathcal{U}^{0}\right)\right]^{-1} \cdot F\left(\lambda, \mathcal{U}^{n}\right) \quad n \geq 0,
$$

or equivalently

$$
D_{\mathscr{U}} F\left(\lambda, \mathcal{U}^{0}\right) \cdot\left(\mathcal{U}^{n+1}-\mathcal{U}^{n}\right)=-F\left(\lambda, \mathcal{U}^{n}\right) .
$$

The drawback of Newton's method is that the stability of its (quadratic) convergence (see [4]) can only be ensured when the first guess $\mathcal{U}^{0}$ is sufficiently near the solution $\mathcal{U}$. If this solution is part of a branch of nonsingular solutions, and if we know the solution at a neighboring point, say $\mathcal{U}(\lambda-\nabla \lambda)$ for an adequate increment $\nabla \lambda$, then we can derive from this value the first guess to start Newton's algorithm.

Since $\lambda \mapsto \mathscr{U}(\lambda)$ is a branch of nonsingular solution of (63), then $F$ is a $\mathscr{C}^{P}$-mapping $(P \geq 2)$, so is the mapping $\mathscr{U}(\lambda)$ and we can differentiate both sides of (63):

$$
\begin{array}{r}
D_{\mathscr{U}} F(\lambda, \mathcal{U}(\lambda)) \cdot\left(\frac{d \mathcal{U}(\lambda)}{d \lambda}\right)+D_{\lambda} F(\lambda, \mathcal{U}(\lambda))=0 \\
\forall \lambda \in \Lambda ;
\end{array}
$$

that is, we find a first order differential equation of the form

$$
\frac{d \mathscr{U}(\lambda)}{d \lambda}=-\phi(\lambda)
$$

where

$$
\phi(\lambda)=\left[D_{\mathcal{U}} F(\lambda, \mathcal{U}(\lambda))\right]^{-1} D_{\lambda} F(\lambda, \mathcal{U}(\lambda)) .
$$

The simplest way to solve (71) is to use the one-step, explicit Euler's method; this brings us to choose

$$
\mathscr{U}^{0}(\lambda)=\mathscr{U}(\lambda-\Delta \lambda)-\phi(\lambda-\Delta \lambda) \Delta \lambda
$$

In other words $\mathcal{U}^{0}(\lambda)$ is defined by

$$
\begin{aligned}
D_{\mathscr{U}} F & (\lambda-\Delta \lambda, \mathcal{U}(\lambda-\Delta \lambda)) \cdot\left(\mathcal{U}^{0}(\lambda)-\mathcal{U}(\lambda-\Delta \lambda)\right) \\
& =-D_{\lambda} F(\lambda-\Delta \lambda, \mathcal{U}(\lambda-\Delta \lambda)) \cdot \Delta \lambda .
\end{aligned}
$$

Let us estimate the difference $\mathscr{U}(\lambda)-\mathcal{U}^{0}(\lambda)$. From (71), we infer that

$$
\mathcal{U}(\lambda)=\mathscr{U}(\lambda-\Delta \lambda)-\int_{\lambda-\Delta \lambda}^{\lambda} \phi(\xi) d \xi
$$

Subtracting (73) from this equality yields

$$
\begin{aligned}
\mathcal{U}(\lambda)-\mathcal{U}^{0}(\lambda) & =-\left[\int_{\lambda-\Delta \lambda}^{\lambda} \phi(\xi) d \xi-\phi(\lambda-\Delta \lambda) \cdot \Delta \lambda\right] \\
& =-\int_{\lambda-\Delta \lambda}^{\lambda} \phi^{\prime}\left(\theta_{\xi}\right) \cdot(\xi-\lambda+\Delta \lambda) d \xi .
\end{aligned}
$$

Hence

$$
\left\|\mathcal{U}(\lambda)-\mathcal{U}^{0}(\lambda)\right\|_{Z} \leq\left[\frac{(\Delta \lambda)^{2}}{2}\right] \max _{\theta \in(\lambda-\Delta \lambda, \lambda)}\left\|\phi^{\prime}(\theta)\right\|_{Z} .
$$

Thus $\left\|\mathscr{U}(\lambda)-\mathcal{U}^{0}(\lambda)\right\|_{Z}$ is $\mathbf{O}\left((\Delta \lambda)^{2}\right)$ and if $\Delta \lambda$ is small enough, the solutions $\mathscr{U}$ and $\mathscr{U}^{0}$ stay close, characterizing the stability, and $\mathcal{U}^{0}$ defined by (73) is an adequate starting value for Newton's algorithm.

\section{Existence Results: Discussion and Concluding Remark}

We have analyzed existence of nonsingular solution to NSEs of type (5), with $\beta=2$, and used Newton's method to study its stability. It happens that the stability of its (quadratic) convergence, when exploiting Newton's method, can only be ensured when the first guess $\mathcal{U}^{0}$ is sufficiently near the solution $\mathcal{U}$. Now what happens for the system (5) with a general $\beta>0$ ? Does a nonsingular solution exist? If yes, in which spaces? We know [19] that there is global existence for the fractional NSEs (5), with only $1 / 2<\beta<1$ and the system is well-posed in some supercritical Besov spaces as well as in the largest critical spaces $\dot{B}_{\infty, \infty}^{-(2 \beta-1)}\left(\mathbb{R}^{n}\right)$. It has also been shown [20] that any classical solution to NSEs (5) with $\beta \geq(1 / 2)+(3 / 4)$ is always global in time. Thus, it is clear that investigations on the system of NSEs of type (5) are far from being complete and the work developed here will contribute to future possible investigation in order to study, for a general parameter $\beta$, the existence and well-posedness of the fractional Navier-Stokes equations in some Banach spaces. 


\section{Conflict of Interests}

The authors declare that there is no conflict of interests regarding the publication of this paper.

\section{References}

[1] B. Mohammadi, Analysis of the K-EPSILON Turbulence, WileyMasson Series, 1997.

[2] R. E. Boisvert, Group analysis of the Navier-Stokes equations [Ph.D Dissertation], Georgia Institute of Technology, Atlanta, Ga, USA, 1982.

[3] R. E. Boisvert, W. F. Ames, and U. N. Srivastava, "Group properties and new solutions of Navier-Stokes equations," Journal of Engineering Mathematics, vol. 17, no. 3, pp. 203-221, 1983.

[4] V. Girault and P. Raviart, Finite Element Methods for NavierStokes Equations, Theory and Algorithms, vol. 5 of Springer Series in Computational Mathematics, Springer, Berlin, Germany, 1986.

[5] D. D. Joseph, Stability of Fluid Motions I, vol. 27 of Springer tracts in Natural Philosophy, Springer, Berlin, Germany, 1976.

[6] J. M. Manale, "Group classification of the two-dimensional Navier-Stokes-type equations," International Journal of NonLinear Mechanics, vol. 35, no. 4, pp. 627-644, 2000.

[7] J. Serrin, "The initial value problem for the Navier-Stokes equations," in Nonlinear Problems, R. E. Langer, Ed., pp. 69-98, University of Wisconsin Press, Madison, Wis, USA, 1963.

[8] A. Atangana and A. Secer, "A note on fractional order derivatives and table of fractional derivatives of some special functions," Abstract and Applied Analysis, vol. 2013, Article ID 279681, 8 pages, 2013.

[9] M. Caputo, "Linear models of dissipation whose Q is almost frequency independent," Journal of the Royal Australian Historical Society, vol. 13, part 2, pp. 529-539, 1967.

[10] E. F. Doungmo Goufo and S. Mugisha, "Mathematical solvability of a Caputo fractional polymer degradation model using further generalized functions," Mathematical Problems in Engineering, vol. 2014, Article ID 392792, 5 pages, 2014.

[11] I. Podlubny, Fractional Differential Equations, Academic Press, San Diego, Calif, USA, 1999.

[12] J. Wu, "Generalized MHD equations," Journal of Differential Equations, vol. 195, no. 2, pp. 284-312, 2003.

[13] A. Kadem and A. Kilicman, "The approximate solution of fractional Fredholm integrodifferential equations by variational iteration and homotopy perturbation methods," Abstract and Applied Analysis, vol. 2012, Article ID 486193, 10 pages, 2012.

[14] G. Jumarie, "On the representation of fractional Brownian motion as an integral with respect to $(\mathrm{d} t)^{a}$," Applied Mathematics Letters, vol. 18, no. 7, pp. 739-748, 2005.

[15] D. Braess, Finite Elements-Theory, Fast Solvers, and Applications in Solid Mechanics, Cambridge University Press, Cambridge, UK, 1997.

[16] V. John and S. Kaya, "A finite element variational multiscale method for the Navier-Stokes equations," SIAM Journal on Scientific Computing, vol. 26, no. 5, pp. 1485-1503, 2005.

[17] J. Leray, "On the motion of viscous liquid filling space," Acta Mathematica, vol. 63, 1934.

[18] M. D. Gunzburger, Finite Element Methods for Viscous Incompressible Flows: A Guide to Theory, Academic Press, London, UK, 1989.
[19] X. Yu and Z. Zhai, "Well-posedness for fractional NavierStokes equations in the largest critical spaces $\dot{B}_{\infty, \infty}^{-(2 \beta-1)}\left(\mathbb{R}^{n}\right)$," Mathematical Methods in the Applied Sciences, vol. 35, no. 6, pp. 676-683, 2012.

[20] J. Wu, “The generalized incompressible Navier-Stokes equations in Besov spaces," Dynamics of Partial Differential Equations, vol. 1, no. 4, pp. 381-400, 2004. 


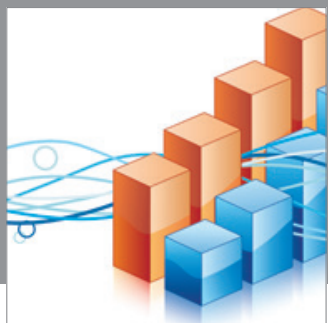

Advances in

Operations Research

mansans

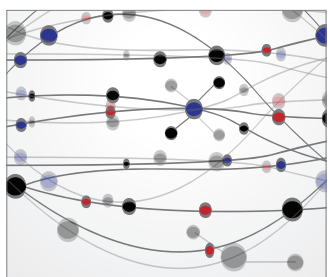

The Scientific World Journal
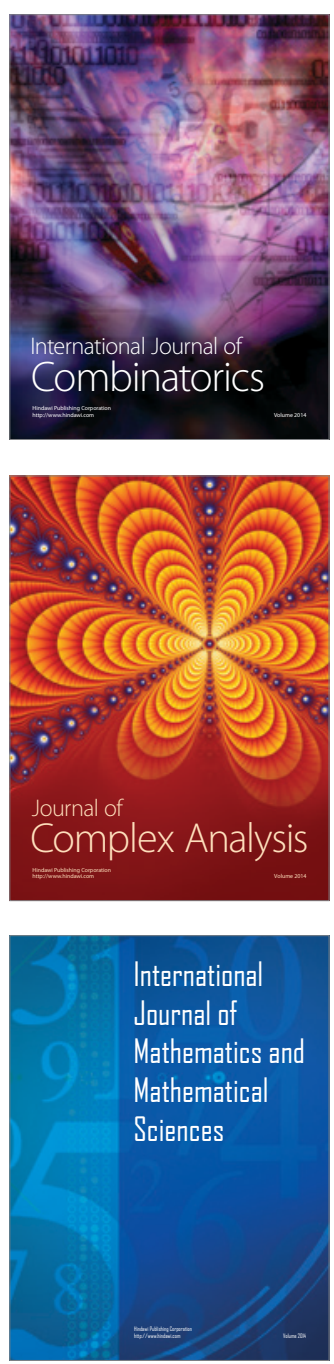
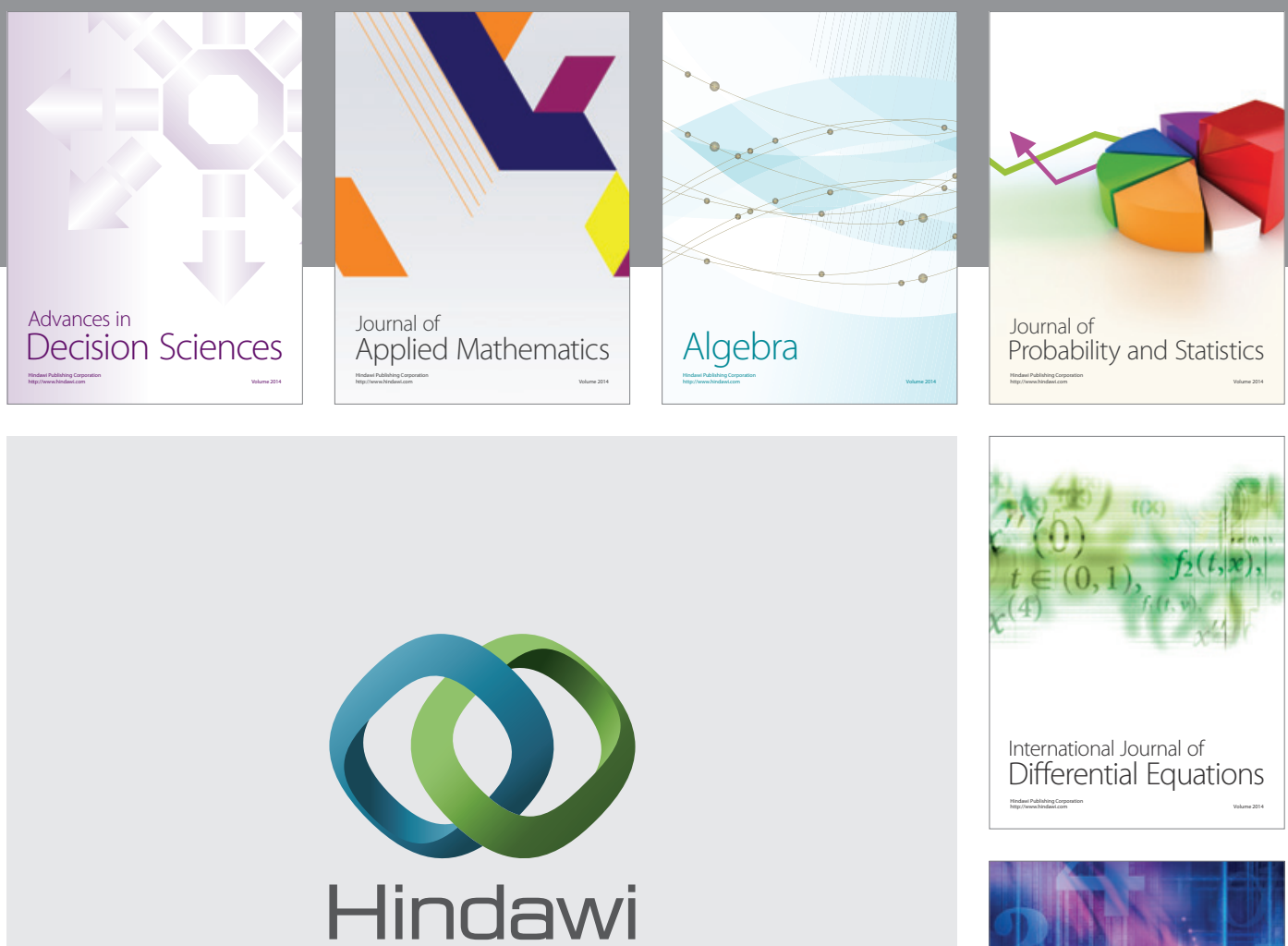

Submit your manuscripts at http://www.hindawi.com
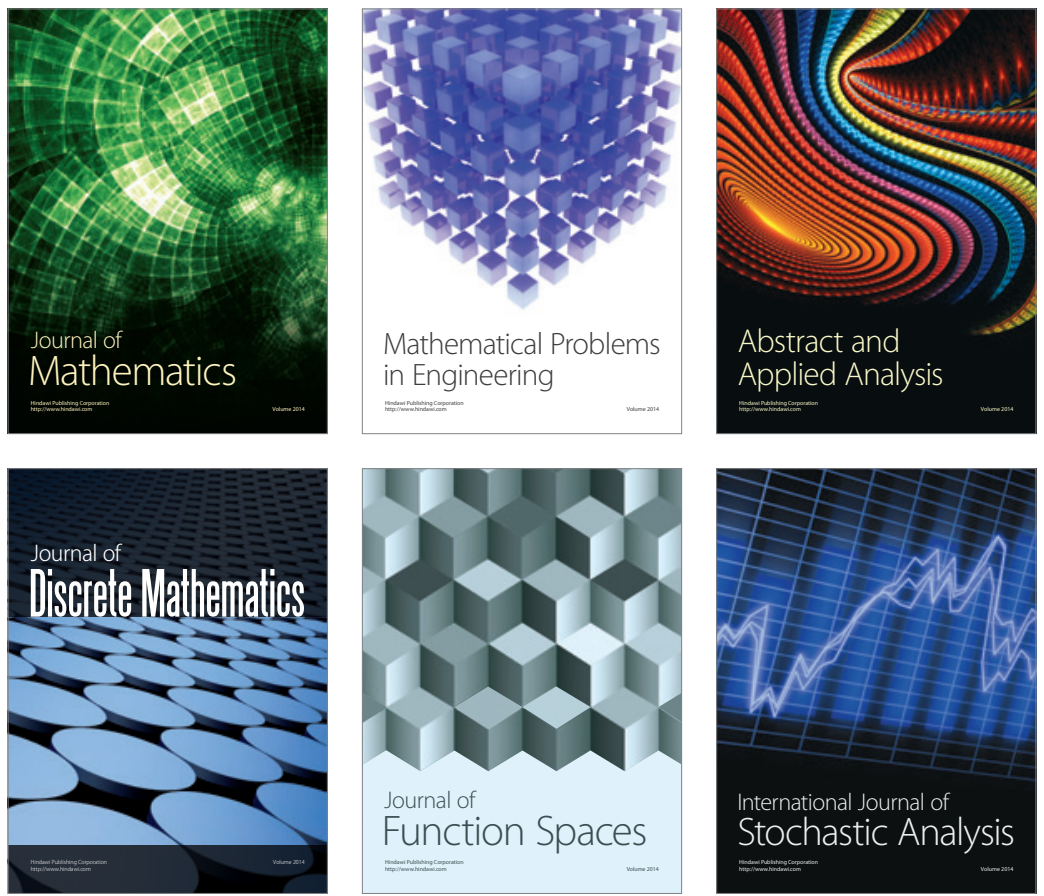

Journal of

Function Spaces

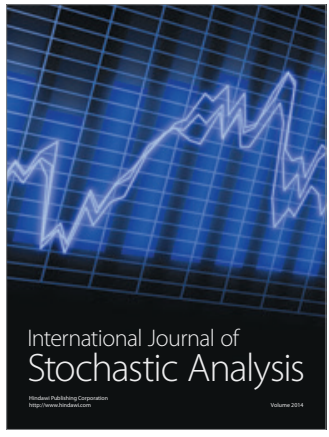

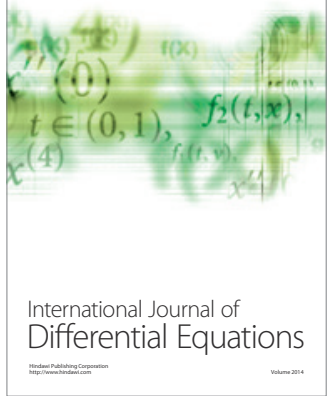
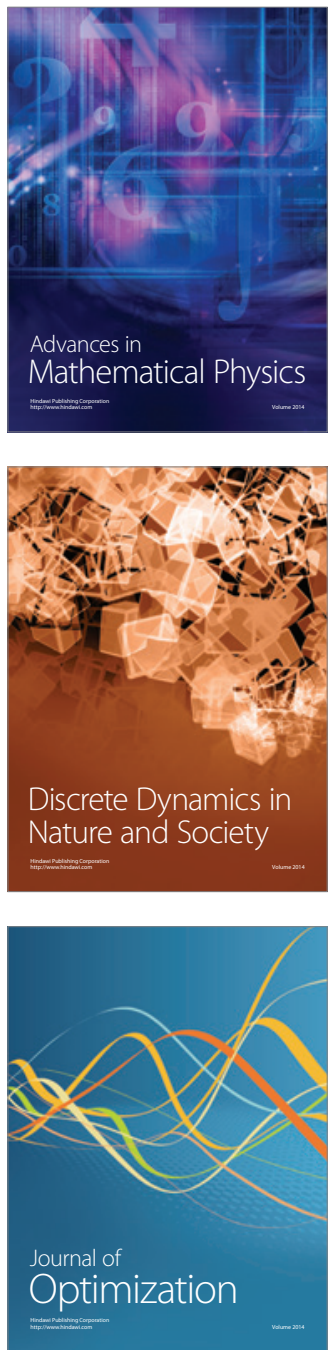